\title{
藏药浴治疗痛风性关节炎 168 例的临床疗效观察
}

Clinical Observation on the Clinical Efficacy of Tibetan Medicine Bath in

\section{Treating 168 Cases of Gout Arthritis}

\section{刘桂梅}

Guimei Liu

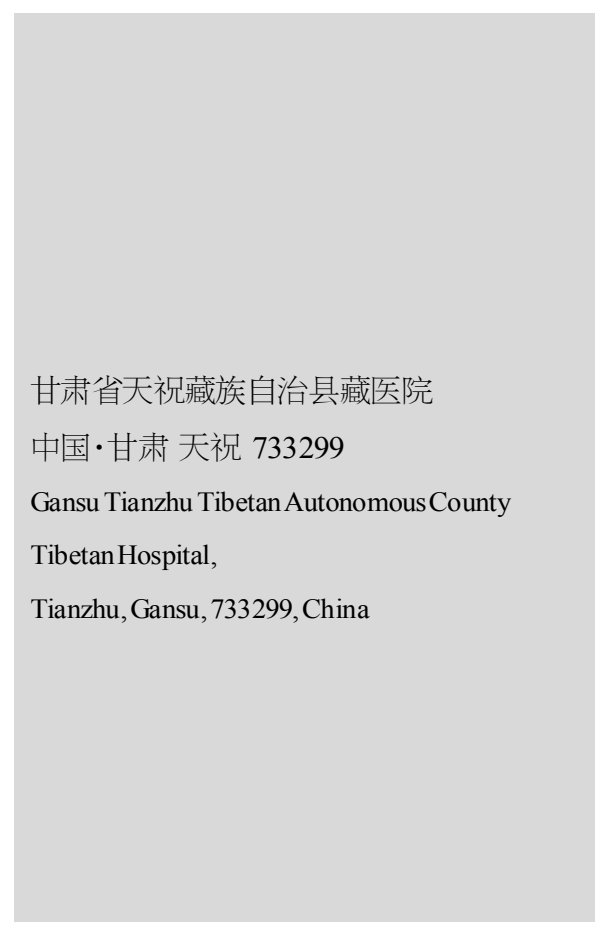

\section{1 引言}

痛风是由于嘌呤代谢紊乱或因尿酸排泄不良导致血尿酸 增加而引起组织损伤的一组疾病。其临床特点为高尿酸血症, 及由此引起的痛风性急性关节炎反复发作, 痛风石沉积, 痛风 性慢性关节炎和关节畸形。本病以关节红、肿、热、痛反复发 作,关节活动不灵活为主要临床表现 ${ }^{[1]}$ 。好发于下肢关节,半数 以上患者首发于拇趾，而在整个病程中约 $90 \%$ 患者大拇趾被 累及。跖趾、踝、膝、指、髋、肘关节为好发部位。尤其拇趾承受 压力最大, 容易损伤, 且局部温度低, 故为痛风性关节炎的部 位。本文以笔者于 2014 年 3 月一2018 年 12 月在甘肃省天祝 县藏医院的临床, 观察 168 例痛风性关节炎患者的住院药浴 疗效,现报告如下。

\section{2 资料与方法}

\section{1 一般资料}

168 例均符合藏医诊断为“直合乃”, 西医诊断为痛风性关 节炎住院患者,全部病例均符合 1977 年美国风湿病学会制定的
诊断标准, 血尿酸值均在 500-600 $\mu \mathrm{mol} / \mathrm{L}$ 。其中, 男性 155 例, 女性 13 例; 年龄 42 67 岁,平均 46.8 岁; 病程 1 14 年, 平均 6.5 年。病情反复发作、红肿热痛发于足拇跖趾关节处 107 例, 发 于踝关节处 42 例, 发于膝关节处 19 例, 均为跖趾关节痛。

\section{2 诊断标准}

\section{2 .1 西医诊断标准}

美国风湿病学会 $(A C R)$ 急性痛风关节炎分类标准, 具备 下列临床、实验室和 $\mathrm{X}$ 线检查 12 项中 6 项者, 或关节液中有 特异性尿酸盐结晶, 或用化学方法或偏振光显微镜证实痛风 石中含尿酸盐结晶, 并除外另一风湿病、骨肿瘤等可确诊为痛 风性关节炎。(1)急性关节炎发作 > 1 次; (2)症反应在 1 天内 达到高峰; (3)单关节炎发作; (4)关节红肿; (5)第一跖趾关节疼 痛或肿胀 ; (6)单侧第一跖趾关节受累 ; (7)单侧趾骨关节受累 ; (8)可疑的痛风石; (9)高尿酸血症; 10X 线片示关节非对称性肿 胀; (11)X 线片示不伴骨质浸润的骨皮质下囊肿; 12关节炎症发 作期间, 关节液微生物培养阴性。

2.2.2 藏医诊断标准

藏医《四部医典》中认为该病早期症状为大腿、腰部、膝关 
临床医学研究 Clinical Medicine Research

节、胫部疼痛生垢、发痒, 活动迟钝、麻木无知觉、沉重。一再发 病, 日益加重,形成痛风。发病初期,病在皮肤和血液之中, 因 而皮肤红肿、灼热、坚硬、有青色斑点疼痛难忍，呵欠频多等诸 多症状, 这时为易治期[2]。若不及时治疗, 蔓延到脉、筋、骨骼、 关节、脂肪等部位时, 严重肿胀, 肿块坚硬灼热, 出现肛门及关 节欲裂等症状, 此时为慢性疾病, 导致瘳腿, 难以治疗。特别是 隆型痛风时有刺痛, 肌肉跳动, 肿势变化不定, 时而散大时而 缩小, 肿胀处时为青玟, 时为黑斑, 肢体僵直, 指趾卷缩, 痛点 用凉物外敷则疼痛略缓解; 赤巴型痛风则症见发热, 肿色赤 红, 痛则拒按; 血型痛风见红肿灼痛, 甚至化脓; 培根型痛风患 部作痒, 沉重而难动, 无知觉。病邪侵入足心时, 拇指结垢呈白 色; 侵入踝部时, 小趾结垢呈黑色; 侵人小腿, 足跟结垢呈花 斑。本病初期阶段为热症, 陈旧则转为寒症。

\section{3 治疗方法}

对 168 例患者均采用低嘌呤、低脂、低糖饮食。控制肉食、 海鲜及豆制品,如米、大豆、蒲公英、奶酪、酸奶、凉开水等性凉 而轻的饮食。禁忌甜食、酸腐、油淢、辛辣之饮食, 禁酒。采用祛 风、除湿、干黄水、止痛消肿、解毒类药物。实施五味甘露药水 浴为主,结合局部放血、艾尒等疗法综合性治疗。

\subsection{1 五味甘露药水浴}

是用山甘露一刺柏叶、草山甘露一杜鹃花、水甘露一柽 柳、阴山甘露一藏麻黄、土甘露一野藁这五味甘露药每份以 $500 \mathrm{~g}$, 依次以 $1: 1: 2: 2: 3$ 的比例配伍。在此基础上对症加减其他 除催吐、下泻、有毒等 3 类药物外的辅助药材而成。对每一位 痛风性关节炎患者, 根据具体病情制定出个性化的药方, 进行 全身浸浴,一般 7 天或 21 天为一疗程,每天 2 3 次,每次持续 时间为 15 30 分钟。并根据患者的病情和浴后的情况掌握其 后续的疗程和药浴的持续时间。

\subsection{2 辅助疗法}

(1) 口服药: 常用五味宽筋藤汤散、十味乳香散、十三味镇痹 散、风湿止痛丸、十八味水银丸、十八味党参丸、二十五味儿茶 丸、二十五味驴血丸、二十五味鬼臼丸、三十五味沉香丸、如意 珍宝丸、二十味沉香丸、五味酎香丸等。药浴开始服用,一直服 用到药浴结束后再坚持一段时间( 1 3 月)。(2)敷法: 是将有刺 激、渗透等作用的药物或者其他物品直接放置在病痛患处及其 周围冷敷或热敷,以便快速解除或减缓病痛的一种医疗方法。

\section{3 疗效观察}

\section{1 疗效标准}

根据第一届全国中西医结合风湿类疾病学术会议修订的 类风湿性关节炎疗效标准进行判定。
显效:用药一个疗程后症状消失或主要症状消除, 各关节 活动功能部分恢复正常, 受累关节肿痛明显好转或消失, $\mathrm{X}$ 线 摄片示软组织肿胀消失, 血沉、类风湿因子滴度降低或已复常;

好转:疼痛症状减轻, 各关节活动功能有所改善;

无效: 与治疗前比较, 受累关节疼痛等各方面均无改善, 甚至加重。

\section{2 疗效分析}

藏医五味甘露汤药效分析: 方中的刺柏叶, 清热解毒, 主 治肾病; 杜鹃花温阳除寒、滋阴益寿、社除一切寒气; 柽柳清毒 热、血热, 治黄水病; 藏麻黄清热解毒, 发汗益疮, 特别能清陈 旧热; 野蕎能止血、消肿、祛风、治疗四肢肿胀。五味配合, 具有 清热解毒、祛风除寒、消肿止痛、滋阴益寿等效能。将上述药物 捣烂煎汤，并根据病情配制加味药物，一起注入浴盆全是浸 浴, 浴后卧热炕发汗。药物配伍煎煮后, 借水温和热炕的物理 作用更能发挥药效, 对治疗痛风、风湿、类风湿性关节炎疗效 更加显著。

\section{3 治疗结果}

通过对 168 例痛风性关节炎患者,经过 1 5 个疗程的藏药 浴综合治疗后, 并经过随访观察。显效 107 例 (63.7\%); 好转 49 例 $(29.2 \%$ ); 无效 12 例 $(7.1 \%$ ), 总有效率为 $92.9 \%$ 。其中, 1 个 疗程后, 显效 16 例; 2 个疗程后, 显效 20 例; 3 个疗程后, 显效 31 例; 4 个疗程后,显效 27 例; 5 个疗程后,显效 13 例。

\section{4 讨论}

藏药浴综合疗法在治疗痛风患者中发现, 其肿痛症状缓 解后, 血尿酸、血沉也随之下降。药浴使皮肤毛细血管扩张, 血 流加速, 促进血液循环及新陈代谢,加速病变周围炎症浸润的 吸收, 起到通络、消肿、止痛的作用 ${ }^{[3]}$ 。临床观察结果表明, 藏药 浴对消除关节疼痛、减低血尿酸和血沉等方面有显著的疗效。

\section{5 结语}

通过 2014 年 3 月一 2018 年 12 月对 168 例痛风关节炎患 者藏药浴治疗的疗效观察, 发现其疗效显著, 不反弹、不复发, 治疗见效快, 简便易行, 治愈率高, 而且经济方便, 在整个治疗 过程中无不良反应, 尤其对社区、基层医院更为实用,值得大 力推广应用。

\section{参考文献}

[1]沈不安.风湿病中医诊治手册[M].北京: 人民军医出版社,2009.

[2]王洁,姚平.三妙散加味治疗痛风关节炎急性发作临床观察 [J]. 中国社区医师(医学专业),2010,12(7): 102 .

[3]贺渊哲,向黎黎,陆小龙,等.骨科消炎酊治疗急性痛风性关节炎 30 例临床观察 $[\mathrm{J}]$.湖南中医杂志,2017,33(7):85-87. 